\title{
B006
}

\section{A 3D Tetrahedral Mesh Generator for Seismic Problems}

\author{
A. Kononov* (Source Contracting), S. Minisini (Shell Global Solutions \\ International BV), E. Zhebel (Shell Global Solutions International BV) \& W. \\ A. Mulder (Shell Global Solutions International BV / TU Delft)
}

\section{SUMMARY}

Finite-element modelling of seismic wave propagation on tetrahedra requires meshes that accurately follow interfaces between impedance contrasts or surface topography and have element sizes proportional to the local velocity. We explain a mesh generation approach by example. Starting from a finite-difference representation of the velocity model, triangulated surfaces are generated along impedance discontinuities. These define subdomains that are meshed independently and in parallel, honouring the local velocity values. The resulting volumetric meshes are merged into a single mesh. The approach is flexible, efficient, scalable and capable of producing quality meshes. 


\section{Copenhagen '12}

\section{Introduction}

3-D seismic wavefield computations with the finite-element method (FEM) are becoming more common nowadays (Komatitsch and Tromp, 1999; Dumbser and Käser, 2006; Zhebel et al., 2011, among many others). This is because finite-difference methods (FDM) are unable to achieve sufficient accuracy in complex geological models for sharp impedance contrasts and for a landscape with, for instance, sand dunes or mountains. For such problems, the FEM, which is able to accurately follow both inner and outer interfaces, has significantly better accuracy at approximately the same or an even lower computational cost (Wang et al., 2010; Moczo et al., 2011).

Explicit time-integration schemes implemented for, e.g., mass-lumped or discontinuous Galerkin FEM schemes (Mulder, 2001; Dumbser and Käser, 2006) impose quite demanding constraints on the quality of the underlying mesh. Not only should interfaces between impedance constrasts be followed accurately by element boundaries, also the diameter of the element should scale with the velocity. A low-quality mesh can severely degrade the efficiency of the FEM approach because of large spatial errors due poor element shapes or elements that are too large. Also, elements that are too small will lead to extremely small time-integration steps and a reduction in performance.

Mesh generators available in structural mechanics are not easily applicable for seismic wavefield computations. For the latter, the main geometric complexity occurs in the interior of a 3-D volume, whereas in structural mechanics, the outer shape of a volume is the primary target of interest, so that the element sizing is completely governed by geometry constraints. There are, however, more similarities to the seismic case in the area of scientific volume visualisation (Zhou et al., 1997; Cignoni et al., 1998, for instance).

The complexity in mesh generation for seismic applications is due to sharp and abruptly varying interfaces and a large modeling volume. Mesh generation becomes even more complex if there is a large number of interfaces and if they have multiple intersections. Moreover, to have the equivalent of a fixed number of points per wavelength as is common with finite differences and to have all elements running at not too small a timestep, the element size should be proportional to the local velocity.

We will explain our tetrahedral mesh generation approach by example, starting from a relatively complex and more or less realistic velocity model specified on a finite-difference grid.

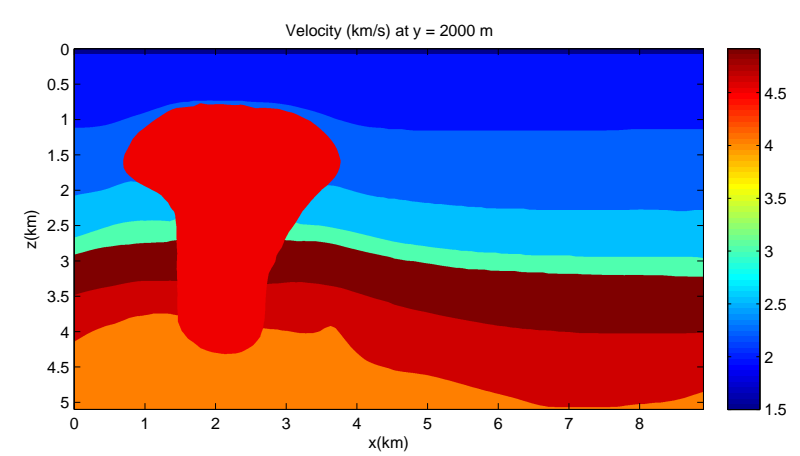

(a)

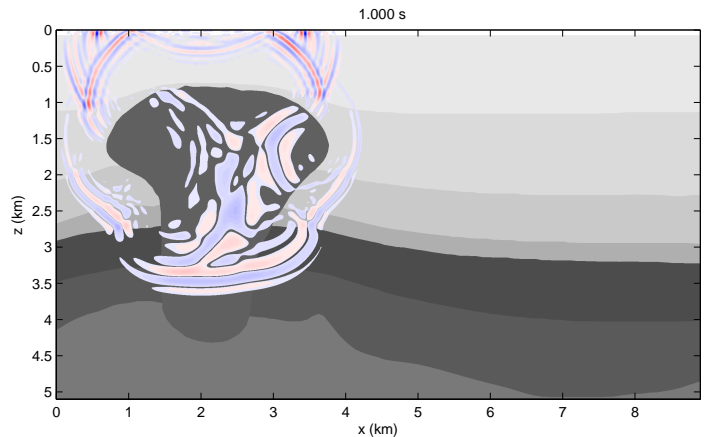

(b)

Figure 1 (a) Vertical cross-section of the 3-D velocity model at $y=2 \mathrm{~km}$. The velocity varies from $1.5 \mathrm{~km} / \mathrm{s}$ in the water layer at the top to $5 \mathrm{~km} / \mathrm{s}$ in a hard layer. (b) Snapshot of the seismic pressure wavefield at 1 s, computed with an acoustic mass-lumped finite-element code (Zhebel et al., 2011).

\section{Method}

The original 3-D velocity model was given on a finite-difference grid with $447 \times 223 \times 256$ points in $(x, y, z)$ and a grid spacing of $20 \mathrm{~m}$, shown in Figure 1(a). A direct application of a mesh generator to the 


\section{Copenhagen '12}

model would result in a poor quality mesh because of the discontinuities in the medium properties. Also, the problem size may lead to inefficiency, because the computational cost of some of the algorithms for mesh improvement and optimization increases rapidly with the number of initial elements, faster than linearly and sometimes even exponentially. This may result in a mesh generator that takes much longer to run than the finite-element computations. The approach described here is able to accomplish the meshing in a reasonably short time.

The first step is the extraction of the interfaces, the surfaces that describe the discontinuities in the velocity model. These are initially represented as point sets in relation to the original finite-difference grid. The interfaces, together with planar faces on the boundaries of the domain, partition the global domain into subdomains that are topologically equivalent to a cube or torus as in the current example, or a body with multiple holes in more complex cases. Note that, prior to the first step, the global domain can be divided into a few blocks in order to accelerate the whole meshing procedure. Secondly, the sampled grid representations of the interfaces are transformed into triangulated surface meshes bounding the subdomains. These should be identical over the shared areas and edges of neighbouring subdomains. Note that step 1 and 2 can be skipped if the initial model is already specified in a surface mesh form, which still may require remeshing.

The third step is to disassemble the model into a number of interconnected volumes, whose connectivity is accomplished via the compatible triangulated surface meshes. As a fourth step, one has to generate volumetric tetrahedral meshes using the surface meshes as input to a 3-D mesh generator, described below. The size of the tetrahedra is controlled by either the velocity fields enclosed in the subdomains or by the surface mesh sizes of nearby interfaces. The fifth step is to merge the meshed subdomains together, producing the final mesh.

This approach has a number of advantages: $(a)$ it can be directly parallelized in straight-forward manner, since each volume and each surface can be meshed independently, thereby reducing the total meshing time; (b) further mesh adjustments and optimization can be applied locally for each subdomain; $(c)$ if the quality of surfaces meshes is good enough, then the overall mesh can be easily refined or coarsened by applying the corresponding operations to the surface and volumetric meshes.

\section{Results}

We will follow the outlined approach to build a mesh for the model shown in Figure 1. The first step, extracting the interfaces, can be accomplished by computing the numerical gradient of the velocity field on the grid and selecting points where it is large. Also, the gradient direction has to be determined in order to select the proper points, i.e., points that actually belong to a particular interface. This can be

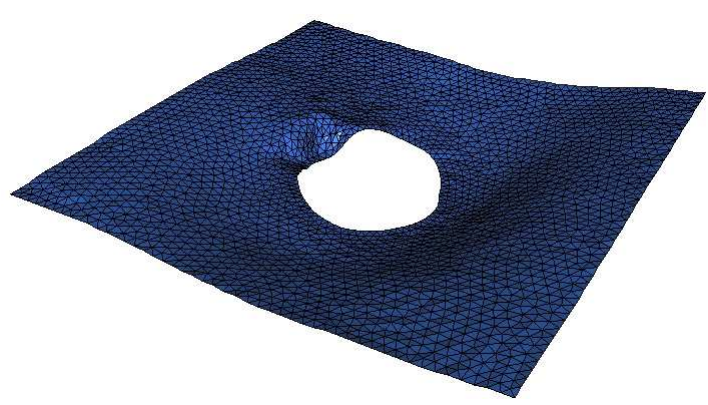

(a)

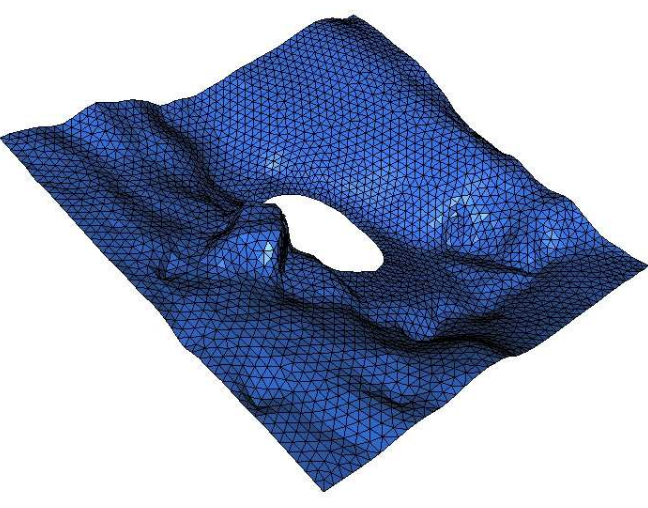

(b)

Figure 2 (a) An example of an extracted interface with a hole due to the intersection with the salt body. (b) Another, deeper interface. 


\section{Copenhagen '12}

quite challenging in areas where interfaces intersect each other, for instance, close to the salt body, the mushroom-shaped object on the left side in Figure 1. In this example, tracing the shape of a salt body was the most complex task, alleviated by the fact that the velocity was a constant $4.5 \mathrm{~km} / \mathrm{s}$ inside the diapir. Seismic interpretation and model building software is, or course, available for that task, providing surface meshes as input, but we wanted to have the additional flexibility handling gridded models. Figure 3(a) shows the extracted salt body. As soon as the shape of an inner inclusion is determined,

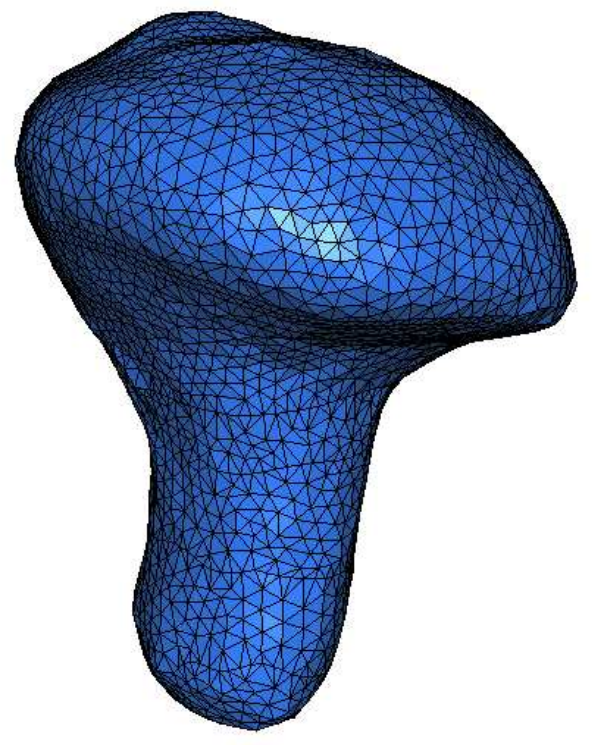

(a)

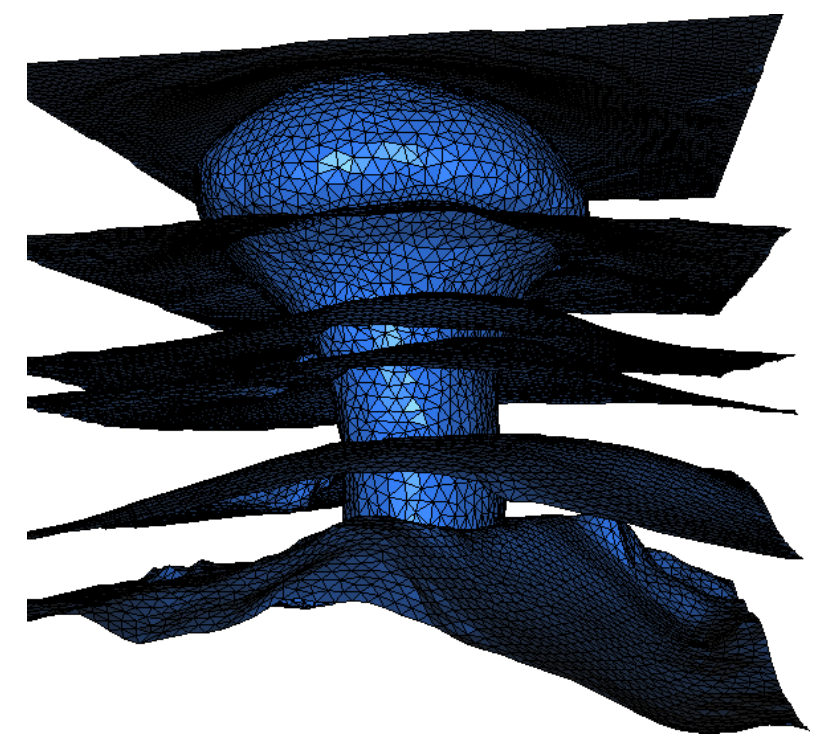

(b)

Figure 3 (a) The salt body shape was extracted and meshed separately. Some of the edges follow the intersection with layer interfaces that still need to be wrapped around the body. (b) Salt body together with the interfaces.

the remaining interfaces can be accurately extracted and meshed as shown in Figures 2 and 3(b). Next, triangulated planar boundary surfaces have to be added for each topological layer. The boundary con-

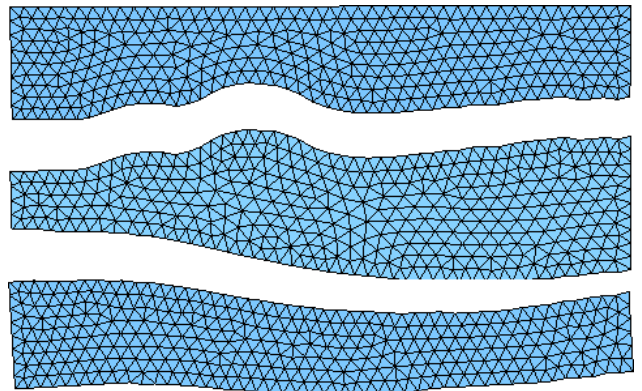

(a)

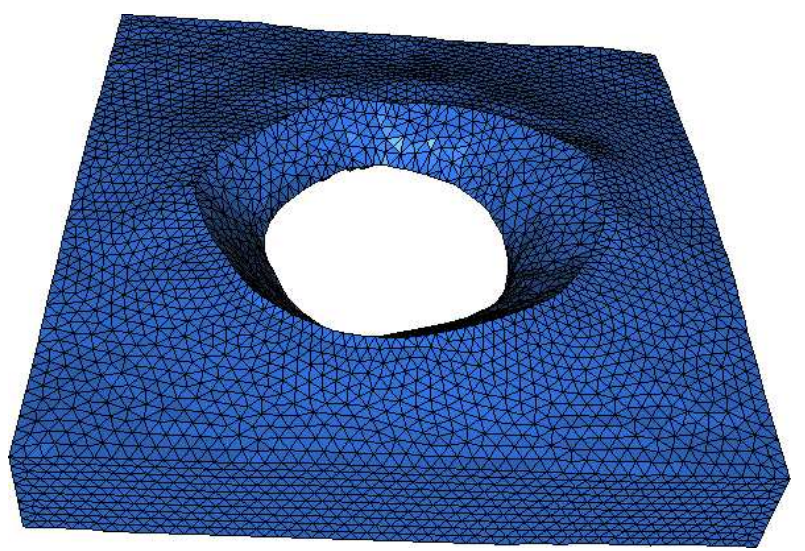

(b)

Figure 4 (a) An example of planar surface meshes, which are forming the sides of neighbouring layers. The matching of the interfaces and the mesh quality can be clearly observed. (b) An example of a closed subdomain that was built by merging the corresponding surface meshes.

tour of each boundary surface consists of two linear vertical segments and undulating curves at top and bottom. The corresponding surface mesh is generated in two steps that have to be iteratively repeated. First, trial points are injected inside the contour according to some heuristic, e.g., moving boundary fronts. Secondly, after the triangulation of the initial point set, a truss system is introduced in which 


\section{Copenhagen '12}

each triangulation edge corresponds to a non-linear spring with an equilibrium length proportional to the local velocity value. A few iterations of such a system towards equilibrium suffice to achieve a good quality surface mesh, as confirmed by Figure 4. When the surface meshing of a subdomain is completed

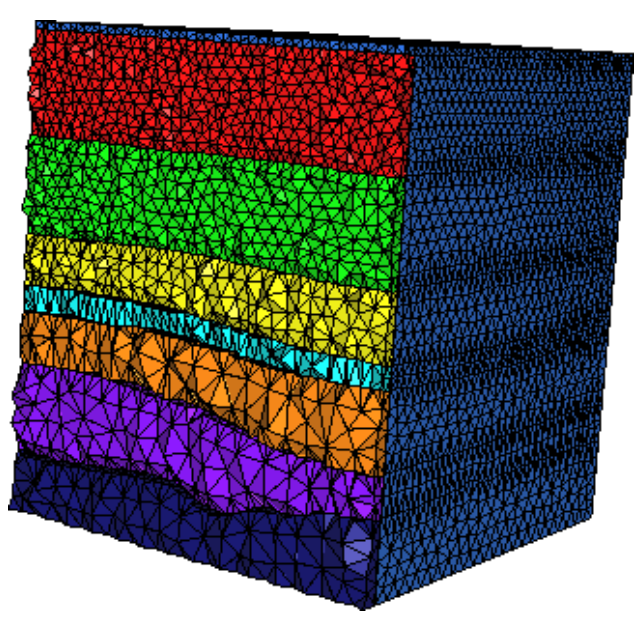

(a)

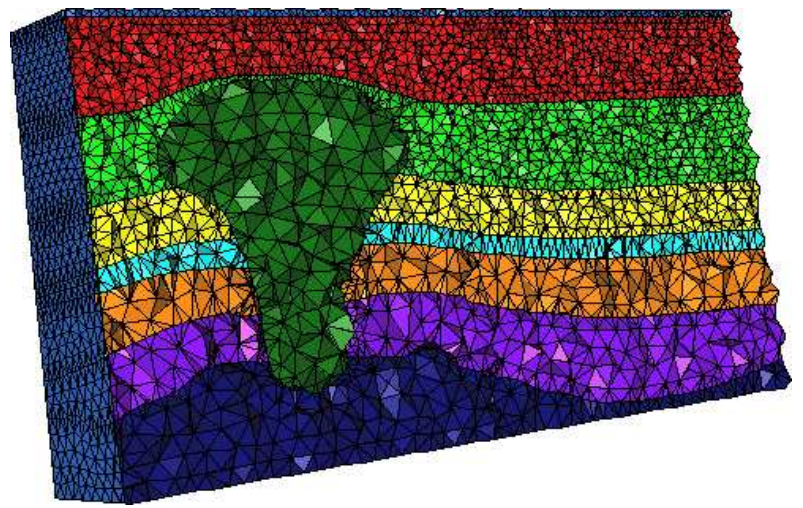

(b)

Figure 5 Two vertical cross-sections of the final mesh: (a) along a yz-plane at some constant $x$ and $(b)$ along an xz-plane at some constant y. Different velocities are marked by different colors. For better visibility, a coarser version of the mesh is displayed.

and assembled, it can be sent as input to a tetrahedral mesh generator that is actually able to handle such input, for example, the mesher by Computing Objects (2010). The final tetrahedral mesh, after merging the subdomain meshes, is shown in Figure 5.

\section{Conclusions}

We have described an approach for 3-D tetrahedral mesh generation and applied it to a non-trivial geological model with large velocity contrasts. Domain decomposition was applied to construct a tetrahedral mesh of good quality, following interface boundaries and having element sizes proportional to the local velocity.

\section{References}

Cignoni, P., Montani, C. and Scopigno, R. [1998] Tetrahedra based volume visualization. In: Hege, H.C. and Polthier, K. (Eds.) Mathematical Visualization - Algorithms, Applications, and Numerics. Springer Verlag, ISBN 3-540-63991-8, 3-18.

Computing Objects [2010] CM2 Meshtools @ . http://www.computing-objects.com.

Dumbser, M. and Käser, M. [2006] An arbitrary high-order Discontinuous Galerkin method for elastic waves on unstructured meshes - II. The three-dimensional isotropic case. Geophysical Journal International, 167(1), 319-336, doi:10.1111/j.1365-246X.2006.03120.x.

Komatitsch, D. and Tromp, J. [1999] Introduction to the spectral-element method for 3-D seismic wave propagation. Geophysical Journal International, 139(3), 806-822, doi:10.1046/j.1365-246x.1999.00967.x.

Moczo, P., Kristek, J., Galis, M., Chaljub, E. and Etienne, V. [2011] 3-D finite-difference, finite-element, discontinuous-Galerkin and spectral-element schemes analysed for their accuracy with respect to Pwave to S-wave speed ratio. Geophysical Journal International, 187(3), 1645-1667, doi:10.1111/j.1365246X.2011.05221.x.

Mulder, W.A. [2001] Higher-order mass-lumped finite elements for the wave equation. Journal of Computational Acoustics, 9(2), 671-680, doi:10.1142/S0218396X0100067X.

Wang, X., Symes, W.W. and Warburton, T. [2010] Comparison of discontinuous Galerkin and finite difference methods for time domain acoustics. SEG Technical Program Expanded Abstracts, 29(1), 3060-3065, doi: 10.1190/1.3513482.

Zhebel, E., Minisini, S., Kononov, A. and Mulder, W.A. [2011] Solving the 3D acoustic wave equation with higher-order mass-lumped tetrahedral finite elements. 73rd EAGE Conference \& Exhibition, Vienna, Austria, Extended Abstracts.

Zhou, Y., Chen, B. and Kaufman, A. [1997] Multiresolution tetrahedral framework for visualizing regular volume data. IEEE Computer Society, IEEE Computer Society, Denver, 3060-3065. 